\title{
Quality Assessment of Oral Anticoagulation by Time in Therapeutic Range in Patients with Venous Thromboembolism in Korea
} \author{
Yang-Ki Kim² \\ 'Depertment of Internal Medicine, Soonchunhyang University Gumi Hospital, Gumi, Korea \\ 2Department of Internal Medicine, Soonchunhyang University Seoul Hospital, Seoul, Korea \\ ${ }^{3}$ Department of Laboratory Medicine, Soonchunhyang University Seoul Hospital, Seoul, Korea \\ ${ }^{4}$ Department of Biostatistics, Korea University College of Medicine, Seoul, Korea
}

Hun-Gyu Hwang, ${ }^{1}$ Myung Shin Kim, ${ }^{1}$ Gune-II Lim, ${ }^{1}$ So-My Koo, ${ }^{2}$ Bo Young Lee, ${ }^{2}$ Jun Yong Chang, ${ }^{2}$ Ki-Up Kim, ${ }^{2}$ Rojin Park, Juneyoung Lee, ${ }^{4}$ Soo-taek Uh,

Received: 10 January 2019 Accepted: 31 January 2019

*Corresponding author: Yang-Ki Kim, MD Department of Internal Medicine, Soonchunhyang University Seoul Hospital, 59 Daesagwan-ro, Yongsangu, Seoul 04401, Korea

Tel: +82-2-709-9114

Fax: +82-2-795-3687

E-mail: kyklung@schmc.ac.kr
Copyright (C) Korean Society on Thrombosis and Hemostasis. All rights reserved.

\begin{abstract}
Purpose: To assess the efficacy and safety of oral anticoagulation with warfarin by time in therapeutic range in patients with venous thromboembolism (VTE) in South Korea.

Methods: This was a retrospective, single-center study to evaluate patients diagnosed with VTE and managed with adjusted dose of warfarin over two-thirds of follow-up period in Thrombosis Clinic between February 2009 and August 2010. Validated dosing algorithm was used to manage the international normalized ratio (INR). Time in therapeutic range was calculated by Rosendaal method.

Results: The period of predetermined warfarin interruption for peri-operative management (1 week preoperatively and 3 weeks post-operatively) was censored. It was not analyzed in 34 episodes. Out of 76 patients with VTE, 47 (23 men; mean age 64.9 years) were analyzed. Approximately two-thirds (31/47) of patients were warfarin-naïve. Total number of admissions was 84 . Expected duration of anticoagulation was 6 months in 22 patients (47\%), 12 months in $1(2 \%)$, and indefinite in $24(51 \%)$. There were various comorbidities, including active malignancy in 7 patients $(15 \%)$, mycobacterium tuberculosis on rifampicin in $4(8.5 \%)$, chronic kidney disease in $4(8.5 \%)$, and hemorrhagic cerebral infarct in $1(2 \%)$. Overall time in therapeutic range (TTR) was $52.4 \%$. Most proportion of out-of-range was below the therapeutic range $(29.5 \%)$. There was no thromboembolic complication, although two cases of major bleeding complications were observed.

Conclusion: The validated dosing algorithm is effective for managing INRs in patients with VTE in Korea.
\end{abstract}

Keywords: Time in therapeutic range, Warfarin, International normalized ratio, Venous thromboembolism

\section{Introduction}

The standard regimen with vitamin $\mathrm{K}$ antagonist (VKA) and lowmolecular-weight heparin (LMWH) was the main therapeutic option in patients with venous thromboembolisms (VTE) until the launch of direct oral anticoagulants (DOAC) in 2013 in South Korea. The ability of health-care provider to make appropriate dosage of VKA (e.g., warfarin) and follow-up decisions can have a major impact on therapeutic effectiveness and safety such as recurrent VTE and bleeding events, respectively. ${ }^{1}$ The quality of anticoagulation can be assessed by time in therapeutic range (TTR). A strong relationship between TTR and bleeding or thromboembolic rates has been observed across studies. ${ }^{2}$ However, TTR in real clinical practice was lower than $57 \%$ to $64 \%$ in clinical trials. ${ }^{3}$ The objective of this study was to investigate the quality of anticoagulation with TTR in patients with VTE in a single tertiary hospital in Korea.

\section{Methods}

Study subjects

This was a retrospective, single-center study to evaluate the adequacy of control for anticoagulation in patients on warfarin to treat VTE. The study population were patients admitted to Soonchunhyang Uni- versity Hospital in Korea between February 2009 and August 2010.

The study protocol was reviewed and approved by the Institutional Review Board (IRB) of the Soonchunhyang University Hospital (IRB No. SCHUH 2018-07-027-001). Informed consent was waived by the IRB because this was a retrospective study.

Seventy-six patients aged 18 years and over who were receiving warfarin therapy for VTE were investigated. They were managed over two thirds of the predetermined follow-up period at Soonchunhyang Thrombosis Clinic.

Patients were excluded if target range of international normalized ratio (INR) was not 2.0-3.0 according to American College of Chest Physicians (ACCP) recommendation. Patients whose test interval between two consecutive INRs exceeded 56 days were also excluded from statistical analysis. The period of predetermined warfarin interruption for peri-operative management (1 week pre-operatively and 3 weeks post-operatively) was censored for 34 episodes. Thus, they were not analyzed. Out of 76, 47 patients who had available data over at least 2 months were included for final analysis.

Dosing algorithm

If the INR was in therapeutic range, there was no change of dose. A $10 \%$ adjustment in the weekly dose of warfarin was used when two consecutive INR results were out of range by no more than 0.5 units 
below or 1.0 unit above the therapeutic INR range while a 10-20\% adjustment in the weekly dose of warfarin was used if the deviation from the therapeutic INR range was more extreme. ${ }^{4}$

TTR was the percent of time in which patients with VTE on warfarin had INR results within the therapeutic range (2.0-3.0) during the test period. The TTR was calculated using linear interpolation method of Rosendaal. ${ }^{5}$

\section{Outcomes}

Primary outcome was TTR. The primary safety outcome was the incidence of major bleeding which was defined as overt bleeding leading to $\mathrm{a} \geq 2 \mathrm{~g} / \mathrm{dL}$ drop in hemoglobin level, transfusion of $\geq 2$ units of packed red blood cells, need for re-operation or invasive intervention, any bleeding at a critical anatomic site (e.g., intracranial, retroperitoneal, intraocular, or pericardial), or fatal bleeding. ${ }^{6}$

Statistical analysis

Continuous variables are presented as mean and range for age while categorical variables are shown as frequencies and percentages.

\section{Results}

Patients characteristics and procedure

Out of 76 patients between February 2009 and August 2010, 47 (23 men; mean age 64.9 years; range, 27-86) were analyzed in this study. Approximately two-thirds (31/47) of these patients were warfarinnaïve. Expected duration of anticoagulation was 6 months in 22 patients (47\%), 12 months in $1(2 \%)$, and indefinite in $24(51 \%)$. There were various comorbidities, including active malignancy in 7 (15\%), mycobacterium tuberculosis on rifampicin in $4(8.5 \%)$, chronic kidney disease in $4(8.5 \%)$, and hemorrhagic cerebral infarct in $1(2 \%)$

Table 1. Baseline characteristics of patients with venous thromboembolism

\begin{tabular}{lc}
\hline Characteristics & Value \\
\hline No. of patients & 47 \\
Age, mean (range) yr & $64.9(27-86)$ \\
Male & $23(49)$ \\
Experience on warfarin & $16(34)$ \\
Expected duration of anticoagulation & \\
6 months & $22(47)$ \\
12 months & $1(2)$ \\
indefinite & $24(51)$ \\
Co-morbidity & \\
Active cancer & $7(15)$ \\
Mycobacterium tuberculosis on rifampicin & $4(8.5)$ \\
Chronic kidney disease & $4(8.5)$ \\
Hemorrhagic cerebral infarct & $1(2)$ \\
\hline
\end{tabular}

Values are presented as number (\%).

Table 2. Distribution according to the value of INR

\begin{tabular}{lcc}
\hline INR & $(\%)$ & Number of counted value \\
\hline$<2.0$ & 29.5 & 3,202 \\
$2.0 \leq \leq 3.0$ & 52.4 & 5,698 \\
$>3.0$ & 18.2 & 1,974 \\
\hline
\end{tabular}

(Table 1). There was no patient whose interval between two consecutive INRs exceeded 56 days.

Overall TTR was $52.4 \%$. Proportions of out-of-range below and above the therapeutic range were $29.5 \%$ and $18.2 \%$, respectively (Table 2). Fig. 1 shows the proportion of patients corresponding to subdivided segment of INR value. The period of predetermined warfarin interruption for peri-operative management (1 week preoperatively and 3 weeks post-operatively) was censored for 34 episodes that were not analyzed. Total number of hospital admission was 84 . There was no thromboembolic complication, although two cases $(4.3 \%)$ of major bleeding complications were observed: a 79-year-old female patient had upper gastrointestinal bleeding on the 4th day of warfarin treatment with concomitant aspirin medication; and a 52-year-old male patient had intramuscular bleeding with high INR of 4.16. All-cause mortality was $0 \%$ ( 0 patient) (Table 3).

\section{Discussion}

This study shows that validated dosing algorithm is effective and safe in getting INRs within therapeutic range for patient with VTE. In this study, overall TTR was $52.4 \%$. This study showed no thromboembolic complication ( $0 \%$ ), although two cases of major bleeding complications (4.3\%) were observed. The incidence of recurrent VTE could be between $4.6 \%$ to $4.9 \%$ based on the study of Veeger et al. ${ }^{7}$ who reported annual incidences of recurrent VTE and major bleeding patients with individual TTR (ITTR) of $45 \%$ to $65 \%$ were similar to those in patient with ITTR of $>65 \%(4.9 \%$ vs. $4.6 \%$ and $2.1 \%$ vs. $1.9 \%$, respectively). Patients with ITTR $<45 \%$ were at higher risk of recurrent VTE than those with ITTR $>65 \%$ (RR 2.8,

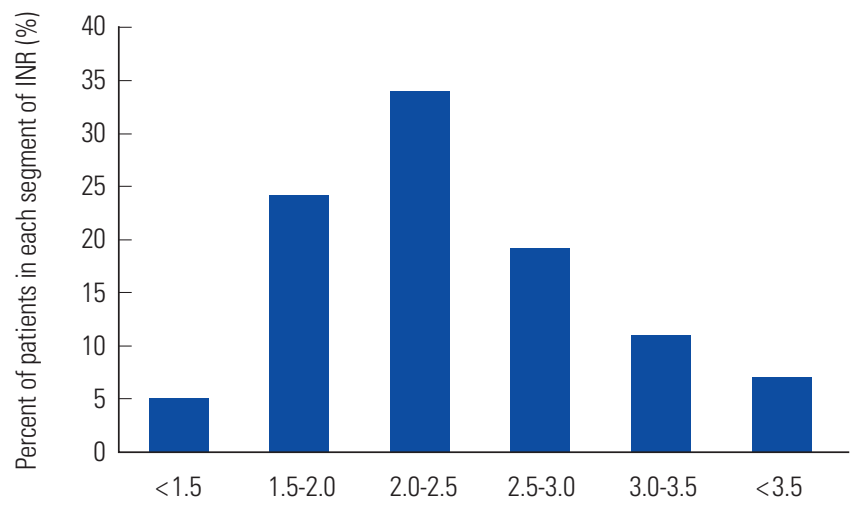

Fig. 1. Proportion of patients corresponding to subdivided segment of INR value.

Table 3. Outcomes of patients with VTE on anticoagulation

\begin{tabular}{lc}
\hline Variables & Value \\
\hline Hospital admission, $\mathrm{n}$ & 84 \\
Bridging anticoagulation, $\mathrm{n}$ & 34 \\
Clinical outcome & \\
Major bleeding & $2(4.3)^{*}$ \\
Thromboembolic complication & $0(0)$ \\
All-cause mortality & $0(0)$ \\
\hline
\end{tabular}

Values are presented as number (\%).

*Two cases of major bleeding complications were observed. 
1.9-4.3, $P<0.001)$.

Walraven et al. ${ }^{8}$ have shown that TTR in patients managed in anticoagulation clinic is $65.6 \%$ compared with $56.7 \%$ in community setting. Manotti et al. ${ }^{9}$ showed that TTR in patients managed with computer-aided dosing system was $71.2 \%$. TTR in thrombosis clinic of Soonchunhyang University Seoul Hospital and usual clinical practice were $52.4 \%$ and $43.7 \%$, respectively. ${ }^{10}$ This study shows that warfarin dosing algorithms are very attractive method to improve the TTR in patients with VTE in Korea. ${ }^{4}$ In this study, the bleeding rate $(4.3 \%)$ was higher than the rate of $2.1 \%$ in patient with ITTR of $45 \%$ to $65 \%$ in the study of Veeger et al. ${ }^{7}$ In subgroup analysis of Hokusai-VTE trial, Nakamura et al. ${ }^{11}$ have suggested that higher risk of bleeding is expected when INR is maintained at 2.0-3.0 given that Japanese guidelines recommend a target INR range of 1.52.5 . $^{12}$ A Chinese study recommended a target INR range of 1.8-2.4. ${ }^{13}$ Korean physicians might tend to maintain lower INR level because of bleeding concerns, considering that $29.5 \%$ were INR $<2.0$ but only $18.2 \%$ were INR $>3.0$ as shown in this study. The optimal target level of INR in Korean population needs to be further evaluated in terms of safety.

This study has some limitations that should be addressed. Firstly, this was retrospective study at a single center. Secondly, the population size of this study was too small $(n=47)$ to generalize results of this study to clinical practice. Thirdly, there was no comparable group regarding anticoagulation management.

This is already the era of DOACs. However there are still patients who need to take VKA in the real world. ${ }^{14}$ Moreover, we have to go back to warfarin to avoid (1) drug interaction with rifampicin, $\mathrm{P}$ glycoprotein, (2) contraindication such as patients with renal failure, or (3) high cost after only 6-month period of insurance in Korea. In this context, this study is very meaningful for patients who have to reach therapeutic INR level in clinical practice.

In conclusion, the validated dosing algorithm is effective for managing INRs in patients with VTE in Korea.

\section{References}

1. Palareti G, Legnani C, Cosmi B, Guazzaloca G, Cini M, Mattarozzi S. Poor anticoagulation quality in the first 3 months after unprovoked venous thromboembolism is a risk factor for long-term recurrence. J Thromb Haemost 2005;3(5):955-61.

2. Ansell J, Hirsh J, Hylek E, Jacobson A, Crowther M, Palareti G. Pharmacology and management of the vitamin K antagonists: American College of Chest Physicians Evidence-Based Clinical Practice Guidelines (8th Edition). Chest 2008;133(6 Suppl):160S-98S.

3. van Es N, Coppens M, Schulman S, Middeldorp S, Buller HR. Direct oral anticoagulants compared with vitamin $\mathrm{K}$ antagonists for acute symptomatic venous thromboembolism: evidence from phase 3 trials. Blood 2014.

4. Kim YK, Nieuwlaat R, Connolly SJ, Schulman S, Meijer K, Raju N, et al. Effect of a simple two-step warfarin dosing algorithm on anticoagulant control as measured by time in therapeutic range: a pilot study. J Thromb Haemost 2010;8(1):101-6.
5. Rosendaal FR, Cannegieter SC, van der Meer FJ, Briet E. A method to determine the optimal intensity of oral anticoagulant therapy. Thromb Haemost 1993;69(3): 236-9.

6. Investigators vG. Idraparinux versus standard therapy for venous thromboembolic disease. N Engl J Med 2007; 2007(357):1094-104.

7. Veeger NJ, Piersma-Wichers M, Tijssen JG, Hillege HL, Meer J. Individual time within target range in patients treated with vitamin $\mathrm{K}$ antagonists: main determinant of quality of anticoagulation and predictor of clinical outcome. A retrospective study of 2300 consecutive patients with venous thromboembolism. British Journal of Haematology 2005;128(4):513-9.

8. van Walraven C, Jennings A, Oake N, Fergusson D, Forster AJ. Effect of study setting on anticoagulation control: a systematic review and metaregression. Chest 2006;129(5):1155-66

9. Manotti C, Moia M, Palareti G, Pengo V, Ria L, Dettori AG. Effect of computer-aided management on the quality of treatment in anticoagulated patients: a prospective, randomized, multicenter trial of APROAT
(Automated PRogram for Oral Anticoagulant Treatment). Haematologica 2001;86(10):1060-70.

10. Samsa GP, Matchar DB, Goldstein LB, Bonito AJ, Lux LJ, Witter DM, et al. Quality of anticoagulation management among patients with atrial fibrillation: results of a review of medical records from 2 communities. Arch Intern Med 2000;160(7):967-73.

11. Nakamura M, Wang YQ, Wang C, Oh D, Yin WH, Kimura $\mathrm{T}$, et al. Efficacy and safety of edoxaban for treatment of venous thromboembolism: a subanalysis of East Asian patients in the Hokusai-VTE trial. J Thromb Haemost 2015;13(9):1606-14

12. Guidelines for the diagnosis, treatment and prevention of pulmonary thromboembolism and deep vein thrombosis (JCS 2009). Circ J 2011;75(5):1258-81.

13. You JH, Chan FW, Wong RS, Cheng G. Is INR between 2.0 and 3.0 the optimal level for Chinese patients on warfarin therapy for moderate-intensity anticoagulation? Br J Clin Pharmacol 2005;59(5):582-7.

14. Hong J, Lee JH, Yhim HY, Choi WI, Bang SM, Lee H, et al. Incidence of venous thromboembolism in Korea from 2009 to 2013. PLoS One 2018;13(1):e0191897. 